\title{
CATHETER ABLATION FOR ATRIAL FIBRILLATION USING THE ABLATION INDEX-HIGH POWER STRATEGY. Do we have the ideal target?
}

\author{
oluwaseun adeola ${ }^{1}$, asad Al Aboud ${ }^{1}$, Travis Richardson ${ }^{1}$, and Gregory Michaud ${ }^{1}$ \\ ${ }^{1}$ Vanderbilt University Medical Center
}

January 12, 2021

\begin{abstract}
Pulmonary vein isolation (PVI) is the cornerstone of catheter ablation for atrial fibrillation (AF) However AF recurrence after a single ablation procedure is common and often attributed to ineffective lesion delivery during PVI. In this issue of the Journal of Cardiovascular Electrophysiology, Chen et al reported their experience with 122 patients who underwent an ablation index-high power (AI-HP) strategy RF ablation for AF using 50W power, targeting AI values of 550 on the anterior left atrium (LA), 400 on the posterior wall and inter-lesion distance (ILD) $6 \mathrm{~mm}$. They achieved 1st pass PVI in $96.7 \%$ of cases, mean RF time was $11.5 \mathrm{~min}$ and total procedure time was only $55.8 \mathrm{~min}$. All patients had $72 \mathrm{~h}$-Holter monitor and trans-telephonic follow up. They reported $89.4 \%$ arrhythmia free survival among patients with paroxysmal AF and $80.4 \%$ among patients with persistent AF at 15-month follow up. Sixty (49\%) patients had luminal esophageal temperature (LET) >390C out of which 3 (2.5\%) had asymptomatic endoscopic esophageal erosions/erythema. Four (3\%) patients had clinically apparent steam pops during ablation with no adverse clinical sequela. While AI-HP guided RF ablation may be an attractive strategy for PVI that likely reduces procedure times and probably has comparable efficacy to conventional ablation settings, its safety requires further evaluation. Feedback from the ablated tissue may need to be incorporated into optimized ablation energy parameters to further improve outcomes.
\end{abstract}

Catheter ablation for atrial fibrillation using the Ablation Index-High power strategy. Do we have the ideal target?

Oluwaseun Adeola MD*, Asad Al Aboud MD*, Travis Richardson MD*, Gregory Michaud MD*

*Arrhythmia Section, Division of Cardiology, Vanderbilt University Medical Center, Nashville, TN

Corresponding Author:

Oluwaseun Adeola, MD

Vanderbilt Heart and Vascular Institute

2220 Pierce Avenue,

383 Preston Research Building,

Nashville, TN 37232, United States

Phone: 857-334-9670

Email: oluwaseun.adeola@vumc.org

Disclosures: none 
Funding: none

Conflicts of interest: none

Pulmonary vein isolation (PVI) is the cornerstone of catheter ablation for atrial fibrillation (AF). Radiofrequency $(\mathrm{RF})$ ablation has evolved significantly over the past two decades with the evolution of 3D electroanatomic mapping, intracardiac echocardiogram (ICE), contact force sensing catheters and catheter irrigation. Nonetheless, the overall freedom from AF after a single ablation is only about $70 \%$ using conventional ablation strategies(1-3). Many AF recurrences can be attributed to incomplete PVI due to ineffective lesion delivery at the index ablation(4).

When performing PVI, the goal of ablation is to create a transmural lesion while avoiding collateral damage to vital structures especially the esophagus given its proximity to the posterior wall of the left atrium. Energy delivered during ablation is converted to heat and causes tissue damage by resistive and conductive heating. Resistive heating occurs when energy delivered from the ablation catheter is converted to heat as it passes through tissue to the ground electrode while conductive heating is due to heat energy being passively transferred from the hot lesion core to the adjacent tissues(5). Several variables have been evaluated in the search for ideal ablation parameters including power and duration of energy application, impedance fall, contact force, catheter stability and inter-lesion distance among others. Ablation index (AI) is a proprietary objective descriptor calculated from contact force, power and duration of a stable catheter position in a weighted formula. AI is independent of impedance fall, though an analysis of 1,013 ablations by Ullah et al showed that AI had a strong correlation with impedance fall(6).

In this issue of the Journal of Cardiovascular Electrophysiology, Chen et al reported their experience with 122 patients who underwent an ablation index-high power (AI-HP) strategy RF ablation for AF using 50W power, targeting AI values of 550 on the anterior left atrium (LA), 400 on the posterior wall and interlesion distance (ILD) $6 \mathrm{~mm}$. They achieved $1^{\text {st }}$ pass PVI in $96.7 \%$ of cases, mean RF time was $11.5 \mathrm{~min}$ and total procedure time was only $55.8 \mathrm{~min}$. All patients had $72 \mathrm{~h}$-Holter monitor and trans-telephonic follow up. They reported $89.4 \%$ arrhythmia free survival among patients with paroxysmal AF and $80.4 \%$ among patients with persistent AF at 15-month follow up. Sixty (49\%) patients had luminal esophageal temperature (LET) $>39^{\circ} \mathrm{C}$ out of which $3(2.5 \%)$ had asymptomatic endoscopic esophageal erosions/erythema. Four $(3 \%)$ patients had clinically apparent steam pops during ablation with no adverse clinical sequela.

Despite the fact that there was no comparison group in this study, their rate of $1^{\text {st }}$ pass PVI is compelling. The paucity of randomized controlled trials on AI guided AF ablation makes it challenging to draw strong conclusions regarding its incremental benefit over conventional strategies. Moreover, what are the best AI targets and ablation power for an ideal ablation lesion? Taghji et al used strict criteria to define lesion depth and contiguity targeting ILD [?]6mm and AI [?]400 on posterior LA and [?]550 on anterior LA defined as the CLOSE protocol while using ablation power $25-35 \mathrm{~W}(7)$. They reported $98 \% 1^{\text {st }}$ pass PVI and $91 \%$ single procedure freedom from atrial tachycardia/atrial fibrillation (AT/AF) at 12 months among 104 consecutive patients with paroxysmal AF off antiarrhythmic drug therapy. Hussein et al showed similar success rates using the same AI targets (400/550) but higher power settings (30-40W) in 89 patients with drug refractory $\mathrm{AF}(8)$. Another study by Solimene et al showed only $10.8 \%$ atrial arrhythmia recurrence among 156 patients with symptomatic AF when the target AI values were 330-350 on LA posterior wall and 400-450 on anterior wall with ablation power 25-35W(9). These studies used different ablation power and AI targets but showed comparable acute success rates to the AI-HP strategy, which appear to be higher than those reported in prior randomized trials using conventional ablation techniques(1-3,10). However, caution should be taken when interpreting success results from small, non-randomized trials. For instance, the AI-HP study appears to have included a population with less advanced AF since the mean LA size and LV function were within the normal range even though $46 \%$ of their patients had persistent atrial fibrillation. Ablation beyond PVI was rare in this study, 17 (13.9\%) underwent cavo-tricuspid isthmus ablation and only 4 patients underwent additional linear LA ablation.

Energy delivered is a product of power and duration of application of that power. Thus, high-power settings 
allow an equivalent amount of energy to be delivered over a shorter duration to achieve defined AI targets or impedance falls, potentially reducing total ablation and procedure times. Indeed, the authors report an impressive mean RF time of $11.5 \mathrm{mins}$ and total procedure time of $55.8 \mathrm{mins}$, essentially less than half the time reported in the CLOSE protocol or the QDOT-FAST trial $(7,11)$. Some of this difference may be explained by the use of deep sedation as opposed to general anesthesia, intracardiac echocardiogram (ICE) was not utilized and assessment of dormant PV conduction was not routinely performed post-ablation.

While high power short duration ablation with prespecified ablation index targets potentially saves time and appears acutely effective, improvement in safety endpoints compared to conventional ablation strategies has not clearly been established. In fact, the authors report a $3 \%(4 / 122)$ incidence of steam pops even though there was no documented pericardial effusion or cardiac tamponade. Much of the energy delivered during ablation is lost to convective cooling from catheter irrigation and surrounding blood pool. Therefore, using standard catheter irrigation settings, ablation using lower power needs to be delivered over a longer duration to allow the endocardium reach target temperatures for irreversible injury. This longer duration allows more time for conductive heating to occur in deeper tissues. Ablation using high power settings (50W) utilizes more resistive heating to create lesions and overcome the endocardial sparing with standard irrigation. This may potentially minimize the time for conductive heating to deeper tissues to occur. However, a significant proportion of patients in this study (49\%) still had LET $>39^{\circ} \mathrm{C}$ although no reported cases of atrio-esophageal fistula occurs and a small fraction (2.5\%) had asymptomatic esophageal erosions/erythema at endoscopy. This would suggest that in the thin posterior LA, high power settings will not somehow violate thermodynamic principles and completely eliminate heating of deeper structures. At the same power, decreasing catheter irrigation rate can allow target endocardial lesion formation to occur over a shorter duration and potentially reduce conductive heat injury to deeper tissues(12). It is also important to note that monitoring esophageal temperature using single thermistors may not accurately portray the risk of esophageal injury especially when these thermistors are far from the ablation site.

The durability of PVI using the AI-HP strategy cannot be assessed based on this study. Eighteen patients had recurrent AT/AF (12 AF, $6 \mathrm{AT}$ ), out of which 9 underwent redo ablation procedures. 5 patients had durable PVI, a rate comparable to prior published studies using conventional ablation strategies(1-3). One must exercise caution in equating freedom for AF with PVI durability since not all patients with reconnected $\mathrm{PVs}$ will manifest recurrent $\mathrm{AT} / \mathrm{AF}$.

The AI-HP strategy only considers ablation energy settings during stable catheter contact but does not include catheter irrigation. It also neglects other important parameters like tissue impedance fall and local electrogram attenuation post ablation which are more reflective of the effects of ablation on the tissue. While AI-HP guided RF ablation may be an attractive strategy for PVI that likely reduces procedure times and probably has comparable efficacy to conventional medium power ablation settings, the incidence of steam pops and continued observation of esophageal heating suggests that the safety of this strategy still requires further evaluation. Feedback from the ablated tissue may need to be incorporated into optimized ablation energy parameters to further improve outcomes, such that lower AI values may be acceptable if steep impedance falls and electrogram attenuation occurs before target AI values are reached.

\section{References}

1. Morillo CA, Verma A, Connolly SJ, Kuck KH, Nair GM, Champagne J, et al. Radiofrequency ablation vs antiarrhythmic drugs as first-line treatment of paroxysmal atrial fibrillation (RAAFT-2) a randomized trial. JAMA - J Am Med Assoc [Internet]. 2014 Feb 19 [cited 2020 Feb 9];311(7):692-9. Available from: http://www.ncbi.nlm.nih.gov/pubmed/24549549

2. Kuck K-H, Albenque J-P, Chun KRJ, Fürnkranz A, Busch M, Elvan A, et al. Repeat Ablation for Atrial Fibrillation Recurrence Post Cryoballoon or Radiofrequency Ablation in the FIRE AND ICE Trial. Circ Arrhythm Electrophysiol [Internet]. 2019 May 22 [cited 2019 Nov 12];12(6):e007247. Available from: http://www.ncbi.nlm.nih.gov/pubmed/31693319

3. Packer DL, Mark DB, Robb RA, Monahan KH, Bahnson TD, Poole JE, et al. Effect of Catheter Ablation 
vs Antiarrhythmic Drug Therapy on Mortality, Stroke, Bleeding, and Cardiac Arrest among Patients with Atrial Fibrillation: The CABANA Randomized Clinical Trial. In: JAMA - Journal of the American Medical Association. American Medical Association; 2019. p. 1261-74.

4. Kuck KH, Hoffmann BA, Ernst S, Wegscheider K, Treszl A, Metzner A, et al. Impact of complete versus incomplete circumferential lines around the pulmonary veins during catheter ablation of paroxysmal atrial fibrillation: Results from the gap-atrial fibrillation-German atrial fibrillation competence network 1 trial. Circ Arrhythmia Electrophysiol [Internet]. 2016 Jan 1 [cited 2020 Dec 30];9(1). Available from: https://www.ahajournals.org/doi/10.1161/CIRCEP.115.003337

5. Kotadia ID, Williams SE, O'Neill M. High-power, short-duration radiofrequency ablation for the treatment of AF. Arrhythmia Electrophysiol Rev [Internet]. 2019 Mar 1 [cited 2020 Dec 25];8(4):265-72. Available from: /pmc/articles/PMC7358956/?report=abstract

6. Ullah W, Hunter RJ, Finlay MC, McLean A, Dhinoja MB, Sporton S, et al. Ablation Index and Surround Flow Catheter Irrigation: Impedance-Based Appraisal in Clinical Ablation. JACC Clin Electrophysiol. 2017;

7. Taghji P, El Haddad M, Phlips T, Wolf M, Knecht S, Vandekerckhove Y, et al. Evaluation of a Strategy Aiming to Enclose the Pulmonary Veins With Contiguous and Optimized Radiofrequency Lesions in Paroxysmal Atrial Fibrillation: A Pilot Study. JACC Clin Electrophysiol. 2018;

8. Hussein A, Das M, Chaturvedi V, Asfour IK, Daryanani N, Morgan M, et al. Prospective use of Ablation Index targets improves clinical outcomes following ablation for atrial fibrillation. J Cardiovasc Electrophysiol [Internet]. 2017 Sep 1 [cited 2020 Dec 27];28(9):1037-47. Available from: https://pubmed.ncbi.nlm.nih.gov/28639728/

9. Solimene F, Schillaci V, Shopova G, Urraro F, Arestia A, Iuliano A, et al. Safety and efficacy of atrial fibrillation ablation guided by Ablation Index module. J Interv Card Electrophysiol. 2019;

10. Kuck K-H, Brugada J, Fürnkranz A, Metzner A, Ouyang F, Chun KRJ, et al. Cryoballoon or Radiofrequency Ablation for Paroxysmal Atrial Fibrillation. N Engl J Med [Internet]. 2016 Jun 9 [cited 2020 Jan 18];374(23):2235-45. Available from: http://www.nejm.org/doi/10.1056/NEJMoa1602014

11. Reddy VY, Grimaldi M, De Potter T, Vijgen JM, Bulava A, Duytschaever MF, et al. Pulmonary Vein Isolation With Very High Power, Short Duration, Temperature-Controlled Lesions: The QDOT-FAST Trial. JACC Clin Electrophysiol. 2019 Jul 1;5(7):778-86.

12. Kumar S, Romero J, Stevenson WG, Foley L, Caulfield R, Fujii A, et al. Impact of Lowering Irrigation Flow Rate on Atrial Lesion Formation in Thin Atrial Tissue: Preliminary Observations From Experimental and Clinical Studies. JACC Clin Electrophysiol [Internet]. 2017 Oct 1 [cited 2021 Jan 4];3(10):1114-25. Available from: https://pubmed.ncbi.nlm.nih.gov/29759494/ 